\title{
POTENCIAL DE RISCO DA TRANSMISSÃO TRANSFUSIONAL DA DOENÇA DE CHAGAS EM BELO HORIZONTE (MG)
}

\author{
Elisabeth Bronfen e Egler Chiari
}

\begin{abstract}
Com o objetivo de se isolar amostras do Trypanosoma cruzi de chagásicos crônicos, foram submetidos a xenodiagnóstico e hemocultura, 59 pacientes provenientes do ambulatório de um dos Hospitais de Belo Horizonte. Esses pacientes informaram, em entrevista prévia, serem ou terem sido doadores de sangue ou candidatos à uma primeira doação. 44 deles $(74,6 \%)$ já haviam doado sangue de 1 a mais de 20 vezes em diferentes bancos de sangue de Belo Horizonte. O resultado conferido atra vés da realização concomitante de apenas 1 xenodiagnóstico e 1 hemocultura, revelou, no grupo de doadores, $47,7 \%$ de positividade parasitológica. Considerando todos os doadores chagásicos crônicos, parasitologicamente comprovados ou não, ocorreram mais de 112 doaf̧ões. Esse é um dado altamente significativo em relação ao problema de transmissão transfusional da doença de Chagas em bancos de sangue de Belo Horizonte, MG.
\end{abstract}

Palavras chaves: Transmissão transfusional. Doença de Chagas. Xenodiagnóstico. Hemocultura.

Os dados apresentados neste trabalho foram obtidos através de um protocolo de entrevista com pacientes chagásicos crônicos. $\mathrm{O}$ nosso contato, com eles, tinha como objetivo submetê-los a xenodiagnóstico e hemocultura para isolamento e amplificação de amostra de Trypanosoma cruzi, dentro de um projetopiloto proposto à Organização Mundial da Saúde.

Esses pacientes estavam sendo acompanhados, clinicamente, em ambulatório de um dos hospitais de Belo Horizonte e relataram ser ou terem sido doadores de sangue na sua maioria, ou candidatos a uma primeira doação nesta cidade.

A amostra foi composta de 59 pacientes pacientes, relativas ao número de doaçôes sangüineas efetuadas, junto com os resultados obtidos através do xenodiagnóstico e hemocultura realizados, caracterizam o risco de transmissão transfusional da doença de Chagas.

\section{CASUÍSTICA E MÉTODOS}

O critério de seleção dos pacientes foi o de terem 3 exames sorológicos concordantes para a doença de Chagas em diferentes laboratórios.

A amostragem ficou composta de 59 pacientes de primeiro atendimento e/ou consulta retorno no ambulatório do Hospital ( 49 homens e 10 mulheres).

A faixa etária compreendeu indivíduos de 20 a 64 anos. O relato quanto ao tempo de moradia em Belo Horizonte variou de 4 a 46 anos.

Centro de Pesquisas "René Rachou" - FIOCRUZ - Belo Horizonte, MG.

Departamento de Parasitologia, Universidade Federal de Minas Gerais.

Suporte financeiro da UNDP/World Bank/WHO/TDR. Recebido para publicaçāo em $8 / 6 / 87$.
Esses pacientes foram então submetidos a 1 xenodiagnóstico com 120 triatomineos de 3 espécies (Panstrongylus megistus, Triatoma infestans e Dipetalogaster maximus) e 1 hemocultura concomitante, colhendo-se $30 \mathrm{ml}$ de sangue venoso heparinizado, com remoçāo do plasma, sendo a seguir os glóbulos semeados $^{2}$ em meio LIT $^{1}$.

\section{RESULTADOS}

Dos 59 pacientes examinados, 44 já haviam doado sangue, e desses, 21 tiveram o xenodiagnóstico e/ou a hemocultura positivos.

A Tabela 1 mostra o número de doações sangüineas efetuadas por 21 pacientes parasitologicamente positivos para o $T$. cruzi; 19 deles estavam positivos no xenodiagnóstico com 1,2 ou 3 espécies de triatomíneos, sendo 7 também positivos na hemocultura e 2 , só na hemocultura.

Esses 21 doadores realizaram um número de 65 doações e por serem parasitologicamente positivos com apenas 1 xenodiagnóstico e 1 hemocultura, aumentaram a chance de ter realmente ocorrido a transmissão via transfusional do $T$. cruzi

A Tabela 2 mostra que 23 pacientes com sorologia positiva para doença de Chagas, mas sem comprovação parasitológica, realizaram um mínimo de 47 doações sangǘneas. Acreditamos que a chance de serem também parasitologicamente positivos, aumentaria se o objetivo do projeto fosse também a realização de mais de um xenodiagnóstico e mais de uma hemocultura por paciente, e/ou os respectivos exames seriados.

Somando-se os dados das Tabelas 1 e 2, referentes ao número de doações, verifica-se que 44 pa- 
Bronfen E, Chiari E. Potencial de risco da transmissão transfusional da doença de Chagas em Belo Horizonte (MG). Revista da Sociedade Brasileira de Medicina Tropical 21:29-32, Jan-Mar, 1988

Tabela 1 - Distribuição das caracteristicas dos 21 doadores de sangue, em Belo Horizonte, sorologicamente positivos para doença de Chagas.

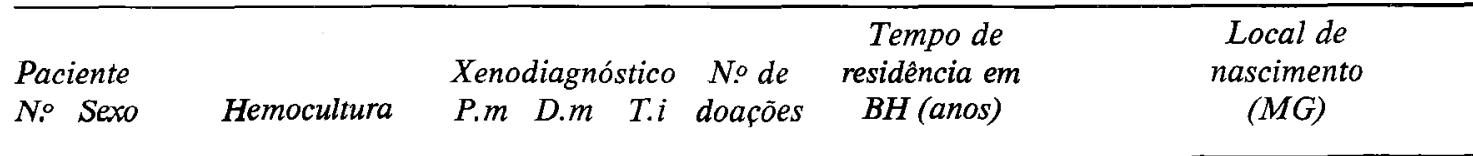

\begin{tabular}{|c|c|c|c|c|c|c|c|c|}
\hline 1 & $F$ & + & + & + & + & 1 & 15 & Deputado Augusto Clementini \\
\hline 2 & $\mathbf{M}$ & - & - & - & + & 1 & 21 & Baldim \\
\hline 3 & M & - & + & - & - & 8 & 12 & Planalto de Minas \\
\hline 4 & $\mathbf{M}$ & - & - & - & + & inúmeras & 17 & Teófillo Otoni \\
\hline 5 & $\mathrm{~F}$ & - & - & + & - & 3 & 26 & Sabinópolis \\
\hline 7 & $\mathbf{M}$ & - & + & + & + & 1 & 4 & Claro das Poções \\
\hline 9 & $\mathrm{M}$ & - & + & + & + & inúmeras & 37 & Belo Horizonte \\
\hline 17 & $\mathrm{~F}$ & + & - & + & + & 1 & 4 & Janaúba \\
\hline 18 & $\mathbf{M}$ & - & - & - & + & 1 & 19 & Diamantina \\
\hline 23 & $\mathbf{M}$ & + & - & - & - & 1 & 15 & Bocaiúva \\
\hline 26 & $\mathbf{M}$ & + & + & + & + & 6 & 24 & Belo Horizonte \\
\hline 28 & $\mathrm{~F}$ & - & + & - & + & 1 & 10 & Buenópolis \\
\hline 30 & $\mathbf{M}$ & + & + & + & - & 1 & 5 & Cedro do Abaeté \\
\hline 31 & $\mathbf{M}$ & - & + & - & - & 3 & 25 & Pequi \\
\hline 38 & $\mathbf{M}$ & - & + & - & - & 1 & 9 & Brasília de Minas \\
\hline 42 & $\mathbf{M}$ & + & - & - & - & 1 & 7 & Chumbo \\
\hline 49 & $\mathbf{F}$ & + & + & + & + & 2 & 31 & Baldim \\
\hline 50 & $\mathbf{M}$ & + & - & + & - & 1 & 46 & Abadia dos Dourados \\
\hline 54 & $\mathbf{M}$ & + & + & + & - & 4 & 9 & Fechados \\
\hline 55 & $\mathrm{M}$ & - & + & - & + & 8 & 47 & Dom Joaquim \\
\hline 57 & $\mathbf{M}$ & - & - & - & + & 20 & 30 & Santa Cruz do Escalvado \\
\hline
\end{tabular}

Panstrongylus magistus $=P . m$

Triatoma infestans $=T . i$

Dipetalogaster maximus $=$ D.m

cientes chagásicos realizaram um número minimo de 112 doações, número esse que exclui 4 pacientes que doaram tantas vezes que não puderam se lembrar do número delas.

A Tabela 3 mostra que, de 15 candidatos a uma primeira doação, recusados com base na sorologia de triagem, 7 foram comprovados parasitologicamente no xenodiagnóstico ou na hemocultura.

\section{DISCUSSÃO}

Segundo Dias \& Brener, a via transfusional da doença de Chegas está se tornando cada vez mais importante na América Latina, devido ao progressivo processo migratório rural urbano e/ao aumento crescente das transfusões sangüineas, junto com a abusiva prática hemoterapêutica 5 .

Os antigos doadores e novos candidatos à doação apresentaram as mesmas caracteristicas parasitológicas, dentro do esperado, para chagásicos crônicos em Minas Gerais, que apresentam uma positividade de aproximadamente $50 \% 26$.
Neste trabalho mostramos 21 doadores chagásicos com xenodiagnóstico e/ou hemocultura positivos para o $T$. cruzi, que realizaram 65 doações sangǘneas. Se o risco teórico minimo de transmissão transfusional da doença de Chagas é de $12,5 \%$ (Dias ${ }^{3}$ ), esses 21 pacientes transmitiram o $T$. cruzi em pelo menos 8 dentre as 65 transfusões. Entretanto, podemos supor que esse grupo de doadores justifique um número muito maior de novos casos de doença de Chagas em Belo Horizonte. Considerando que dois dos fatores de "risco" sejam a parasitemia do doador e o número de doações sangüineas efetuadas ${ }^{34}$, um deles, com apenas a hemocultura positiva e que realizou 20 doações, pode ter sido, sozinho, a fonte de aparecimento de 5 novos chagásicos.

Coura (apud Dias ${ }^{5}$ ) estimou que existem cerca de 90.000 chagásicos vivendo em Belo Horizonte e, dessa forma, os 44 casos aqui apresentados e as 112 doações sangüineas por eles efetuadas indicam apenas uma pequena parcela do problema da transmissão transfusional da doença de Chagas. 
Bronfen E, Chiari E. Potencial de risco da transmissão transfusional da doença de Chagas em Belo Horizonte (MG). Revista da Sociedade Brasileira de Medicina Tropical 21:29-32, Jan-Mar, 1988

Tabela 2 - Número de doações sangüíneas efetuadas por 23 doadores de sangue, em Belo Horizonte, sorologicamente positivos para doenças de Chagas.

\begin{tabular}{rrrcl}
\hline $\begin{array}{l}\text { Paciente } \\
N o\end{array}$ & Sexo & $\begin{array}{c}\text { No de } \\
\text { doaçóes } \\
\text { feitas }\end{array}$ & $\begin{array}{c}\text { Tempo de } \\
\text { residência em } \\
\text { BH (anos) }\end{array}$ & \multicolumn{1}{c}{$\begin{array}{c}\text { Local de } \\
\text { nascimento }\end{array}$} \\
\hline 6 & M & inúmeras & 47 & São Gonçalo do Abaeté \\
10 & M & 7 & 23 & Abaeté \\
15 & M & 2 & 13 & Planalto de Minas \\
16 & M & 4 & 12 & Dom Silvério \\
19 & M & 2 & 6 & Porteirinha \\
22 & M & 2 & 11 & Santana de Pirapã \\
24 & M & 3 & 26 & Florestal \\
29 & M & 2 & 18 & Serro \\
32 & M & 1 & 7 & Planalto de Minas \\
33 & M & 1 & 7 & Pequi \\
36 & M & inúmeras & 59 & Jetibá \\
41 & M & 1 & 10 & Santa Rita \\
43 & M & 1 & 8 & Carbonita \\
44 & M & 2 & 10 & Corinto \\
45 & M & 1 & 9 & São Francisco \\
46 & M & 2 & 31 & Biquinhas \\
47 & M & 2 & 19 & Belo Horizonte \\
48 & M & 1 & 8 & Desembargador Otoni \\
51 & M & 1 & 5 & Mangalos \\
52 & M & 3 & 32 & Venda Nova \\
53 & M & 6 & 8 & Pains \\
56 & F & 1 & 13 & Mantena \\
59 & M & 2 & Carmésia \\
\hline
\end{tabular}

Tabela 3 - Perfil parasitológico de 15 candidatos a doadores de sangue, em Belo Horizonte, serologicamente positivos para doença de Chagas.

\begin{tabular}{lll}
\hline Paciente & \multicolumn{2}{c}{ Xenodiagnóstico } \\
No Sexo Hemocultura P.m D.m T.i
\end{tabular}

\begin{tabular}{rlllll}
\hline 8 & M & - & - & - & - \\
11 & M & - & - & - & - \\
12 & M & - & - & - & - \\
13 & M & - & - & - & - \\
14 & M & - & - & - & + \\
20 & M & - & + & - & - \\
21 & M & - & - & - & - \\
25 & F & - & - & - & - \\
27 & F & - & - & - & - \\
34 & M & + & + & - & - \\
35 & M & - & + & - & - \\
37 & M & + & - & - & - \\
39 & M & + & - & - & - \\
40 & F & + & - & - & - \\
58 & M & - & & - & - \\
& & & & &
\end{tabular}

Panstrongylus megistus $=P . m$

Triatoma infestans $=T . i$

Dipetalogaster maximus $=D . m$

\section{SUMMARY}

Fifty nine chronic chagasic individuals from a out-patient clinics in Belo Horizonte, who use to act as blood donors were submitted to xenodiagnosis and hemocultures for isolating Trypanosoma cruzi. Forty four of them (74.5\%) had already given blood 1 to 20 times. The results, based on only one xenodiagnosis and one blood culture per patient revealed $47.7 \%$ of parasitological positivity. The whole population studied wes responsible for 112 donations. These data stress the importance of transmission of Chagas disease through blood transfusion in Belo Horizonte.

Key words: Transfusional transmission. Chagas' disease. Xenodiagnosis. Hemoculture.

\section{AGRADECIMENTOS}

Ao Afonso da Costa Viana, Orlando Carlos Magno e Oswaldo de Souza Morais pelo trabalho técnico de laboratório.

\section{REFERÊNCIAS BIBLIOGRÁFICAS}

1. Camargo EP.Growth and differentiation in Trypanosoma cruzi. I. Origin of metacyclic trypanosomes in liquid 
Bronfen E, Chiari E. Potencial de risco da transmissão transfusional da doença de Chagas em Belo Horizonte (MG). Revista da Sociedade Brasileira de Medicina Tropical 21:29-32, Jan-Mar, 1988

media. Revista do Instituto de Medicina Tropical de São Paúlo, 6:93-100, 1964.

2. Chiari E, Dias JCP, Lana M, Chiari CA. Hemocultures for parasitological diagnosis of human chronic Chagas' disease (Em publicação).

3. Dias JCP. Mecanismos de transmissão. In: Brener Z, Andrade Z, (ed.) O Trypanosoma cruzi e a doença de chagas.

Guanabara Koogan Ed.: Rio de Janeiro. p. 152-174, 1979.
4. Dias JCP. Enfermedad de Chagas en la region urbana de Belo Horizonte, Minas Gerais, Brasil. Conferência. Reunión Enfermedad Chagas. Córdoba, Argentina, 1984.

5. Dias JCP, Brener Z. Chagas' disease and blood transfusion. Memórias do Instituto Oswaldo Cruz, 79 (suppl.): 139-147, 1984.

6. Krettli AU, Cançado JR, Brener Z. Criterion of cure of human Chagas' Disease after specific chemoterapy: recent advances. Memórias do Instituto Oswaldo Cruz 79 (suppl.): 157, 1984. 